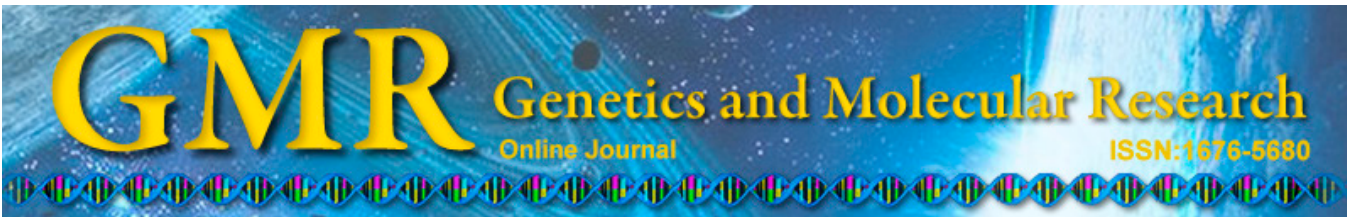

\title{
Replication timing regulation in adults with chromosomal balance rearrangements
}

\author{
Y. Xie ${ }^{1,2}$, J. Wu ${ }^{2}$, S. Lin ${ }^{2}$, Y. Zhou ${ }^{2}$, Q. Fang ${ }^{2}$ and X. Sun ${ }^{1}$ \\ ${ }^{1}$ Key Laboratory of Major Obstetrics Diseases of Guangdong Province, \\ Key Laboratories on Reproductive and Genetic of Guangdong, \\ The Third Affiliated Hospital of Guangzhou Medical University, Guangzhou, \\ China \\ ${ }^{2}$ Fetal Medicine Center, The First Affiliated Hospital of Sun Yat-sen University, \\ Guangzhou, China
}

Corresponding author: X. Sun

E-mail: xiaofangsun@yahoo.com

Genet. Mol. Res. 14 (3): 7833-7840 (2015)

Received November 6, 2014

Accepted March 5, 2015

Published July 14, 2015

DOI http://dx.doi.org/10.4238/2015.July.14.9

\begin{abstract}
The alternative forms of the alleles in biallelic genes display a synchronous pattern of replication that is different from genes subjected to monoallelic expression, which exhibit an asynchronous mode of replication. The present study sought to gain insight into changes in the allele-specific replication timing in phenotypically normal humans with balanced chromosomal rearrangements, and to investigate the potential mechanism for chromosomal rearrangements. We used fluorescence in situ hybridization and chose biallelic gene expression of $R B 1$ and monoallelic expression of $S N R P N$ in phytohemagglutininstimulated lymphocytes to compare differences in the allelic replication timing. We found that compared to genetically normal adult controls, adults with a normal phenotype despite chromosomal rearrangements showed normal replication timing (synchronous or asynchronous) for the $R B 1$ gene and $S N R P N$ genes. Our data support a link between chromosomal aberrations and epigenetic stability in phenotypically normal humans, independent of the breakpoints in chromosomal
\end{abstract}


structural disruption, and represent an epigenetic mark for allelic exclusion in balanced chromosomal rearrangements in patients with normal phenotypes.

Key words: Chromosomal rearrangements; Epigenetic profile; Fluorescence in situ hybridization; Replication timing

\section{INTRODUCTION}

Differences in the DNA replication period of the cell cycle (S phase) can reflect the inheritance of the main genetic characteristics (Gilbert, 2002; McNairn and Gilbert, 2003). Active genes generally replicate earlier than silent genes (Benacerraf, 1987; Gilbert, 2002). A typical example of this phenomenon is provided by the two X chromosomes in mammals, where the silenced X chromosome replicates later than the active one (Priest et al., 1967). Replication timing and epigenetic silencing also relate to synchronous biallelic replication and asynchronous monoallelic replication (Kitsberg et al., 1993; Goldmit and Bergman, 2004).

Phytohemagglutinin (PHA) is a mitogen that stimulates the transition of lymphocytes from G0 to mitosis. In normal circumstances, mitotic figures are not found in human peripheral blood. PHA can promote the abundant growth of mitotically active cells in vitro, allowing an evaluation of the DNA replication period. Replication timing is easily assayed by fluorescence in situ hybridization (FISH) (Boggs and Chinault, 1997) analysis of interphase nuclei. FISH reveals a single dot (singlet; S) for an unreplicated locus and a double signal for a replicated locus (doublet; D). Thus, cells exhibit patterns signaling synchronicity (SS cells or DD cells) or asynchronicity (SD cells). A high frequency of SD cells indicates an asynchronous mode of replication, and a low frequency reveals a synchronous mode. For estimation of the frequency of SD cells, at least 100 cells with two clear signals were scored from each sample for each locus, following one-color FISH with the relevant probe. Without the use of 5-bromo-2'-deoxyuridine to identify the $\mathrm{S}$ phase, this FISH assay can be used to measure replication timing (Boggs and Chinault, 1997; Dotan et al., 2008). Application of this method has shown that monoallelically expressed genes undergo asynchronous replication, and actively expressed genes that are expressed from two alleles undergo earlier DNA replication (Knoll et al., 1994; Gunaratne et al., 1995; Simon et al., 1999). Based on Mendelian genetics, mammals receive two alleles of each gene from their parents. Both alleles are regulated symmetrically with respect to expression activity, and replication is synchronous, such as in the case of the genes TP53, RB1, AML1, HER2, and C-MYC (Hiratani and Gilbert, 2009; Grinberg-Rashi et al., 2010). However, monoallelically expressed genes are also found in the genome, in which the replication of the DNA of the two alleles is asynchronous, such as in SNRPN (Hiratani and Gilbert, 2009; Grinberg-Rashi et al., 2010).

A number of studies have shown the known monoallelically expressed genes are nonsynchronous (Hiratani and Gilbert, 2009; Grinberg-Rashi et al., 2010). These genes include the following examples: 1) imprinted genes, particularly the Prader-Willi/Angelman syndrome gene $S N R P N$; the paternal allele replicates earlier than the maternal allele (Knoll et al., 1994; Gunarathe et al., 1995; Simon et al., 1999); 2) genes on the inactive X chromosome in females (Boggs and Chinault, 1994); and 3) exclusively biallelic genes (Mostoslavsky et al., 2001). Therefore, the replication timing of a gene (synchronous or asynchronous) is considered to be an epigenetic characteristic (Gribnau et al., 2003; Ensminger and Chess, 2004; Gimelbrant et al., 2005). 
The $R B 1$ gene in the peripheral blood lymphocytes of patients with $22 \mathrm{q} 11.2$ microdeletion syndrome and 7q11.23 microdeletion syndrome, which normally replicate synchronously, lose their synchronous mode and replicate asynchronously (Yeshaya et al., 2009). This phenomenon suggests that chromosome abnormalities lead to abnormal phenotypes and disrupted genetic stability. The diploid DNA conformation is crucial for epigenetic stability and some aspects of the function of the gene (Parada and Misteli, 2002).

In this study, we were curious to know whether there were differences in replication timing between adults with a normal phenotype and a normal chromosome number $(\mathrm{CN})$ and adults with a normal phenotype with balanced chromosomal rearrangements (NCR).

\section{MATERIAL AND METHODS}

\section{Study subjects}

Ten subjects were NCR and 14 subjects were CN (Table 1).

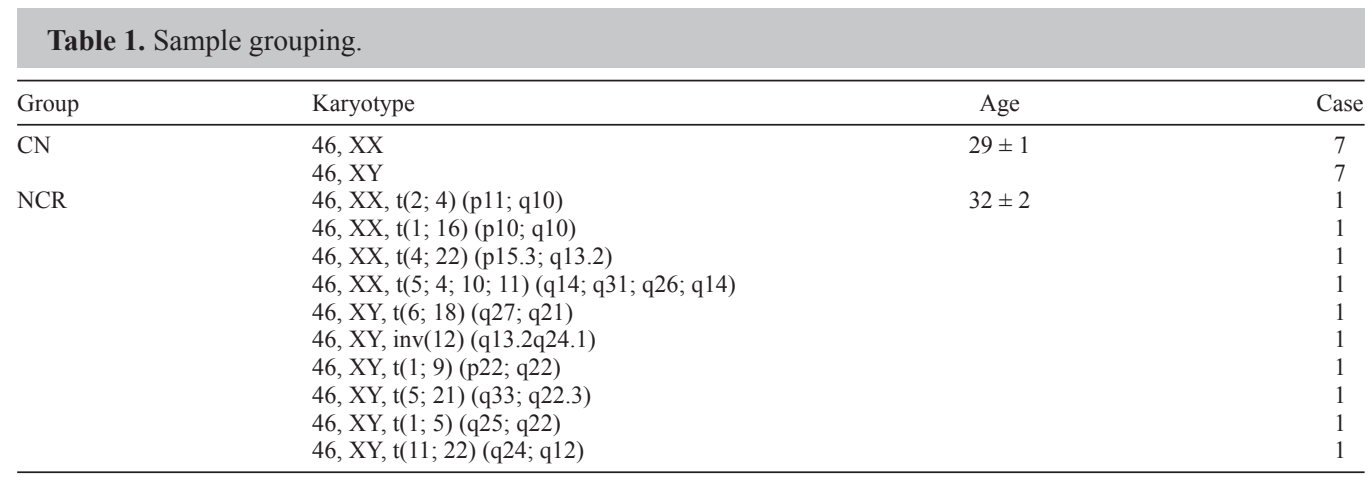

$\mathrm{CN}$ : control group, adults with a normal phenotype and a normal chromosome number; NCR: adults with a normal phenotype with balanced chromosomal rearrangements.

\section{Lymphocyte cultures}

Each adult donated $2 \mathrm{~mL}$ peripheral blood prior to medical treatment. The cell cultures of PHA-stimulated lymphocytes were prepared according to the standard protocol used for routine karyotype assays (Dotan et al., 2004). The culture medium contained $20 \%$ fetal calf serum, $3 \%$ PHA, $0.2 \%$ heparin, and $1 \%$ antibiotics (penicillin/streptomycin). The cultures were incubated at $37^{\circ} \mathrm{C}$ for $72 \mathrm{~h}$ followed by another $3 \mathrm{~h}$ after colcemid (Sigma, Israel; final concentration: $0.08 \mu \mathrm{g} / \mathrm{mL})$ was added. Then, hypotonic treatment $\left(0.075 \mathrm{M} \mathrm{KCl}\right.$ at $37^{\circ} \mathrm{C}$ for $\left.30 \mathrm{~min}\right)$ and fixation (methanol:acetic acid solution $=3: 1$, freshly prepared and pre-cooled) were performed. The cell suspensions in the fixative solution were stored at $-20^{\circ} \mathrm{C}$ until required for analysis by FISH.

\section{DNA probes}

Two directly labeled commercial probes obtained from Vysis, Inc. (Downers Grove, 
IL, USA) were used: i) the $S N R P N$ probe (Vysis 05J26-027), which identifies the imprinted $S N R P N$ gene, located on the long arm of chromosome 15 (15q11-q13, within the Prader-Willi/ Angelman syndrome imprinted region) adjacent to the centromere, the probe was also premixed with a CEP15 control probe; and ii) the $R B 1$ probe (Vysis 32-190001), which identifies the retinoblastoma tumor suppressor gene, a classical biallelically expressed gene (Benacerraf et al., 1987).

\section{Probe application}

Each of the 24 samples was hybridized individually by two-color FISH with the $S N R P N$ and $R B 1$ probes.

\section{FISH}

FISH was performed according to the protocol described in the literature (Dotan et al., 2004). Briefly, cells were dropped onto two-well slides without pretreatment. Diluted probe solution $(5 \mu \mathrm{L})$ was added to the targeted area of the sample slides. The slides were then covered with round 18 -mm silanized coverslips, and sealed with rubber cement. They were placed in a micro-heating system (Vysis, Inc.) that was programmed for 3 min denaturation at $73^{\circ} \mathrm{C}$ followed by $18 \mathrm{~h}$ of hybridization at $42^{\circ} \mathrm{C}$. The control probe was hybridized simultaneously.

\section{Post-hybridization treatments}

After the coverslips were removed, the post-hybridization washes consisted of immersing the slides for $2 \mathrm{~min}$ in a solution of $0.4 \mathrm{X}$ sodium chloride and sodium citrate (SSC) (Abbott Laboratories, Abbott Park, IL, USA), pH 7.0, with 0.3\% Nonidet-40 (NP-40, a nonionic detergent) at $75^{\circ} \mathrm{C}$, followed by $30 \mathrm{~s}$ in $2 \mathrm{X} \mathrm{SSC}$ with $0.1 \% \mathrm{NP}-40$ at room temperature in a shaking water bath. After brief drying, the slides were coated with an antifade solution containing 4,6-diamidino-2-phenylindole ( $0.125 \mu \mathrm{g} / \mathrm{mL}$; Vysis, Inc.), covered with glass coverslips, and stored at $-20^{\circ} \mathrm{C}$ until further analysis.

\section{Cytogenetic evaluation}

The slides were analyzed blindly by two skilled technicians using an Olympus BX51 fluorescent microscope equipped with a triple band-pass filter. The FISH replication assay was used to estimate the replication status of each allelic region, as described previously (Dotan et al., 2004). At least 100 interphase nuclei were analyzed in each sample. The high SD-cell frequency indicated an asynchronous mode of replication, and the low frequency indicated a synchronous mode (Patsalis et al., 2004).

\section{Statistical method}

The statistical significance of the differences between two cell populations was determined using the two-tailed Student $t$-test (Microsoft Excel), and a P value $<0.05$ was considered to be statistically significant (analysis of variance, least significant difference $t$-test). 


\section{Ethical basis}

Informed consent was obtained from each individual examined, and the Ethics Committee of the First Affiliated Hospital of Sun Yat-sen University approved the study.

\section{RESULTS}

To determine whether there were differences of replication timing between $\mathrm{CN}$ and NCR adults, a FISH analysis assay was carried out with the human peripheral blood lymphocytes from the samples, as listed in Table 1 (NCR, 10 cases; CN, 14 cases). Figure 1 shows the representative FISH images of SS cells (Figure 1A), SD cells (Figure 1B), and DD cells (Figure 1C). In a population of replicating cells, the frequency of SD cells of the total population of cells with two fluorescent signals represented the level of asynchrony in the replication timing of the identified alleles. Figure 2 shows the full view of the ratios of SS, SD, or DD cells for $R B 1$ and $S N R P N$ genes in the subjects in the $\mathrm{CN}$ group (Figure $2 \mathrm{~A}$ and $\mathrm{B}$ ) and in the NCR group (Figure $2 \mathrm{C}$ and $\mathrm{D}$ ). In summary, the difference in the ratio of SD cells between the CN and NCR groups was not statistically significant (CN: $20.53 \pm 5.48 \%$ vs NCR: 17.88 $\pm 6.00 \%, \mathrm{P}>0.05$ ) (Figure 3).
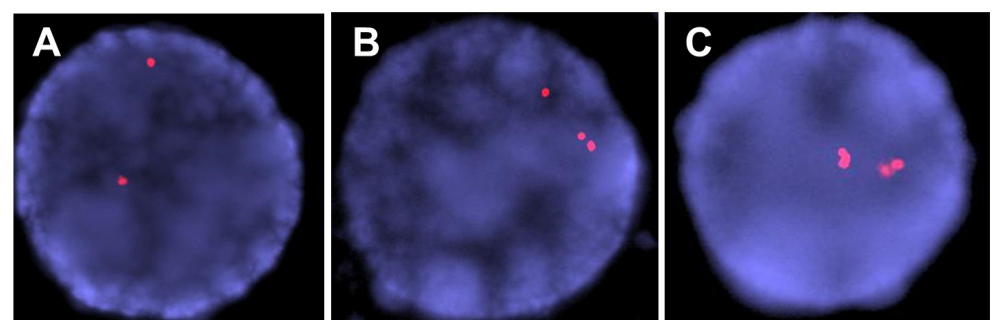

Figure 1. Fluorescence signals in phytohemagglutinin-stimulated lymphocytes after fluorescence in situ hybridization with $R B 1$ probes. A. Cell with 2 singlets (SS cell) representing cells in which both alleles have not yet replicated. B. Cell with 1 singlet and 1 doublet (SD cell) representing S-phase cells where only 1 of the allelic sequences has replicated. C. Cell with 2 doublets (DD cell) representing cells in which both alleles have replicated.
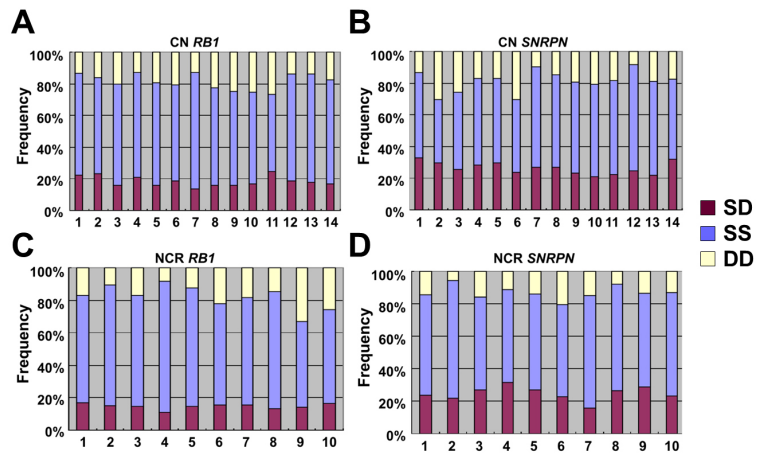

Figure 2. Mean frequencies of SS cells, SD cells, and DD cells for the $R B 1$ or $S N R P N$ loci in the 14 subjects of the $\mathrm{CN}$ group (adults with a normal phenotype and a normal chromosome number) and the 10 subjects of the NCR group (adults with a normal phenotype with balanced chromosomal rearrangements). A. $R B 1$ in the CN group. B. $S N R P N$ in the CN group. C. $R B 1$ in the NCR group. D. SNRPN in the NCR group. 


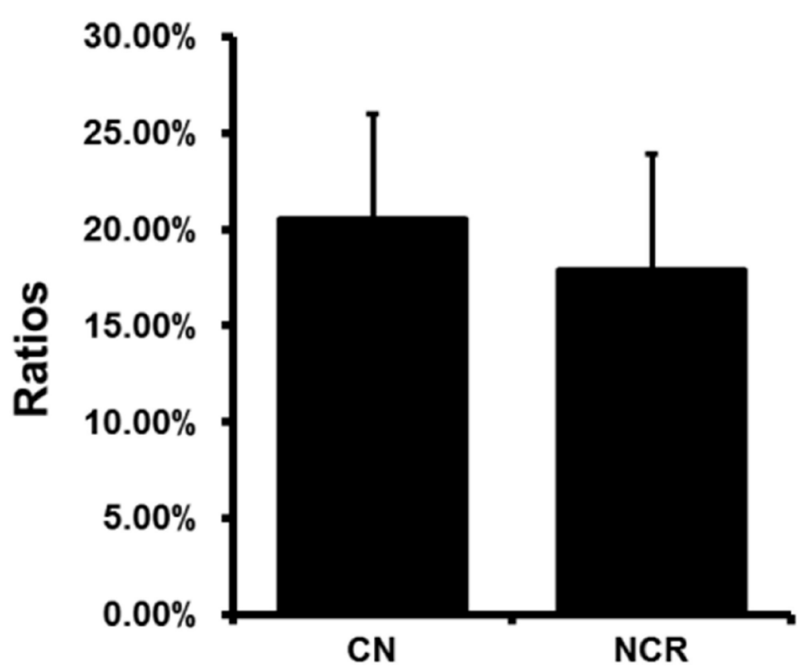

Figure 3. Comparison of the $\mathrm{SD}$ cells between the two groups. $\mathrm{N}=3, \mathrm{P}>0.5$.

\section{DISCUSSION}

Chromosomes are influenced by physical, chemical, biological, and genetic factors, leading to structural distortions, followed by breaks and reunion. If the broken fragments rejoin in their original positions, there may be no genetic effects. If they do not, rearrangements occur, such as chromosomal deletion, duplication, and translocation, amongst others (Wincent et al., 2010).

Carriers of balanced chromosomal rearrangements retain the total number of genes, but the expression of genes that are dependent on chromosomal position changes, leading to functional changes and changes in the individual phenotype. In this study, we compared adults with balanced chromosomal rearrangements and a normal phenotype with a normal control group. The ratio of the SD cells, indicating asynchronous replication, showed no statistical difference.

In a study of 22q11.2 microdeletion syndrome, which has several presentations including DiGeorge syndrome and velocardiofacial syndrome, and 7q11.23 microdeletion syndrome (Williams syndrome), Yeshaya et al. (2009) found that compared with normal samples, the frequency of patients' SD cells for the RBI and SNRPN genes retained the change. This indicates that an imbalanced rearrangement of the chromosomes may cause an epigenetic change, which leads to alteration of the phenotype. Moreover, in keeping with the present study, the individuals with balanced chromosomal rearrangement did not present a change.

As summarized by Hiratani and Gilbert (2009) the link between replication timing and chromatin structure is still unclear, with indirect evidence supporting two rather intuitive genres of models in which early replication dictates open chromatin or open chromatin dictates early replication. Previous studies have confirmed that the change in the timing of DNA replication involves epigenetic regulations, such as methylamination (Parada and Misteli, 2002; Ensminger and Chess, 2004), phosphorylation (Smith et al., 2001), or deacetylation (Demeret et al., 2001).

The difference in the timing of the replication of the two alleles is due to methylation (Parada and Misteli, 2002; Ensminger and Chess, 2004). It is unclear whether there is an independent mechanism regulating methylamine levels between the synchronously and asynchronously replicating alleles. The control of the timing of allele replication in chromosome 
microdeletion patients may be due to microdeletions accompanied by changes in the level of allelic methylation. Smith et al. (2001) found that certain chromosome translocations exhibit a delay in mitotic chromosome condensation that is associated with a delay in the mitosisspecific phosphorylation of histone $\mathrm{H} 3$ in the peripheral blood lymphocytes of breast cancer patients. They considered the possible reason for this effect to be the deletion and/or rearrangement of one arm of the affected chromosome, leading to the deletion or mutation of a cis element that normally establishes early replication timing, resulting in delayed replication of the entire chromosome. Demeret et al. (2001) also reviewed chromatin remodeling complexes featuring histone deacetylase activity, and found that such complexes may be involved in DNA replication by embedding origins of replication into a repressive, deacetylated chromatin structure, which would reduce their accessibility to replication factors, delaying the timing of DNA duplication during the S phase, as observed for heterochromatin.

Hence, the results of the present study suggest that a normally balanced chromosomal rearrangement is not accompanied by changes in the epigenetic regulation status, or an abnormal epigenetic change can be corrected through other pathways to restore the allele replication timing. Therefore, such rearrangements do not influence the phenotype.

According to our results, the normal phenotype of a balanced chromosomal rearrangement may also maintain the normal function of the genes and their epigenetic stability. Our findings supplement the theory of the relationships between normal phenotype and DNA replication timing; they will inform the debate on aristogenesis, and will be useful in the formulation of a biomarker for prenatal diagnosis of normal fetal phenotype.

\section{Conflicts of interest}

The authors declare no conflict of interest.

\section{ACKNOWLEDGMENTS}

We would like to thank the Clinical Cytogenetics Laboratory for aiding in the data collection. We are also grateful to the individuals included in this study as well as their families. Research supported by the National Natural Science Foundation-Guangdong Joint Fund (\#U1132005, \#31171229), the Science and Information Technology of Guangzhou Key Project (\#2011Y1-00038, \#201400000004), the Guangdong Province Higher Education Funding (\#Yq2013135), and the National Natural Science Foundation of China (\#31171229) to X.F. Sun.

\section{REFERENCES}

Benacerraf BR, Gelman R and Frigoletto FD Jr (1987). Sonographic identification of second-trimester fetuses with Down's syndrome. N. Engl. J. Med. 317: 1371-1376.

Boggs BA and Chinault AC (1994). Analysis of replication timing properties of human X-chromosomal loci by fluorescence in situ hybridization. Proc. Natl. Acad. Sci. U. S. A. 91: 6083-6087.

Boggs BA and Chinault AC (1997). Analysis of DNA replication by fluorescence in situ hybridization. Methods 13: 259270.

Demeret C, Vassetzky Y and Méchali M (2001). Chromatin remodelling and DNA replication: from nucleosomes to loop domains. Oncogene 20: 3086-3093.

Dotan ZA, Dotan A, Ramon J and Avivi L (2004). Altered mode of allelic replication accompanied by aneuploidy in peripheral blood lymphocytes of prostate cancer patients. Int. J. Cancer 111: 60-66. 
Dotan ZA, Dotan A, Ramon J and Avivi L (2008). Aberrant allele-specific replication, independent of parental origin, in blood cells of cancer patients. BMC Cancer 8: 390.

Ensminger AW and Chess A (2004). Coordinated replication timing of monoallelically expressed genes along human autosomes. Hum. Mol. Genet. 13: 651-658.

Gilbert DM (2002). Replication timing and transcriptional control: beyond cause and effect. Curr. Opin. Cell. Biol. 14: 377-383.

Gimelbrant AA, Ensminger AW, Qi P, Zucker J, et al. (2005). Monoallelic expression and asynchronous replication of p120 catenin in mouse and human cells. J. Biol. Chem. 280: 1354-1359.

Goldmit M and Bergman Y (2004). Monoallelic gene expression: a repertoire of recurrent themes. Immunol. Rev. 200: 197-214.

Gribnau J, Hochedlinger K, Hata K, Li E, et al. (2003). Asynchronous replication timing of imprinted loci is independent of DNA methylation, but consistent with differential subnuclear localization. Genes. Dev. 17: 759-773.

Grinberg-Rashi H, Cytron S, Gelman-Kohan Z, Litmanovitch T, et al. (2010). Replication timing aberrations and aneuploidy in peripheral blood lymphocytes of breast cancer patients. Neoplasia 12: 668-674.

Gunaratne PH, Nakao M, Ledbetter DH, Sutcliffe JS, et al. (1995). Tissue-specific and allele-specific replication timing control in the imprinted human Prader-Willi syndrome region. Genes Dev. 9: 808-820.

Hiratani I and Gilbert DM (2009). Replication timing as an epigenetic mark. Epigenetics 4: 93-97.

Kitsberg D, Selig S, Brandeis M, Simon I, et al. (1993). Allele-specific replication timing of imprinted gene regions. Nature 364: 459-463.

Knoll JH, Cheng SD and Lalande M (1994). Allele specificity of DNA replication timing in the Angelman/Prader-Willi syndrome imprinted chromosomal region. Nat. Genet. 6: 41-46.

McNairn AJ and Gilbert DM (2003). Epigenomic replication: linking epigenetics to DNA replication. Bioessays 25: 647656.

Mostoslavsky R, Singh N, Tenzen T, Goldmit M, et al. (2001). Asynchronous replication and allelic exclusion in the immune system. Nature 414: 221-225.

Parada L and Misteli T (2002). Chromosome positioning in the interphase nucleus. Trends Cell. Biol. 12: 425-432.

Patsalis PC, Evangelidou P, Charalambous S and Sismani C (2004). Fluorescence in situ hybridization characterization of apparently balanced translocation reveals cryptic complex chromosomal rearrangements with unexpected level of complexity. Eur. J. Hum. Genet. 12: 647-653.

Priest JH, Heady JE and Priest RE (1967). Delayed onset of replication of human X chromosomes. J. Cell Biol. 35: 483487.

Simon I, Tenzen T, Reubinoff BE, Hillman D, et al. (1999). Asynchronous replication of imprinted genes is established in the gametes and maintained during development. Nature 401: 929-932.

Smith L, Plug A and Thayer M (2001). Delayed replication timing leads to delayed mitotic chromosome condensation and chromosomal instability of chromosome translocations. Proc. Natl. Acad. Sci. U. S. A. 98: 13300-13305.

Wincent J, Bruno DL, van Bon BW, Bremer A, et al. (2010). Sixteen New Cases Contributing to the Characterization of Patients with Distal 22q11.2 Microduplications. Mol. Syndromol. 1: 246-254.

Yeshaya J, Amir I, Rimon A, Freedman J, et al. (2009). Microdeletion syndromes disclose replication timing alterations of genes unrelated to the missing DNA. Mol. Cytogenet. 2: 11. 